\title{
Estudo do comportamento da difusividade térmica do aço inoxidável duplex UNS S32304 submetido a processo de soldagem TIG, em função do tratamento térmico
}

\author{
Caetano, G. A. ${ }^{1}$; Souza, N. S. ${ }^{1}$; Orlando, M. T. D. ${ }^{1}$; Luz, T. S. ${ }^{1}$ \\ 1 Departamento de Engenharia Mecânica, Universidade Federal do Espírito Santo, Vitória, ES, Brasil.
}

\begin{abstract}
Resumo
O foco deste trabalho é o estudo do material candidato a revestimento externo dos receptáculos de contenção para transporte de substâncias que sejam fontes de alta radioatividade, em especial ${ }^{99} \mathrm{Mo}$, para a cadeia produtiva associada as indústrias radiofarmacêuticas. Em especial relatamos os efeitos dos processos de soldagem TIG e tratamento térmico na difusividade térmica de amostras de um aço inoxidável duplex UNS S32304, faz-se necessário o conhecimento das propriedades desse material frente ao processo de fabricação com propósito de atender as normas de segurança de transportes de materiais radioativos. As amostras foram soldadas em pares seguindo um procedimento autógeno, utilizando argônio e nitrogênio como gás de proteção. Em seguida os pares soldados foram tratados termicamente à temperatura de $600^{\circ} \mathrm{C}$ seguido de resfriamento de uma em ar e a outra em água do mar. $\mathrm{A}$ análise da difusividade térmica foi realizada com uso do método Flash Laser após as amostras serem submetidas ao processo de soldagem e posterior tratamento térmico e a caracterização microestrutural por microscopia óptica. As regiões soldadas apresentaram valores de difusividade térmica superiores aos encontrados no metal base das amostras, o que pode ter sido influenciado pelo aumento do tamanho dos grãos nessas regiões e o desequilíbrio de fases.
\end{abstract}

\begin{abstract}
The potential material to be the outer covering of the contention receptacles with the goal of transporting highly radioactive substances, specially ${ }^{99} \mathrm{Mo}$, to the radiopharmaceutical industries network, is the main focus of this study. Where the effects are reported of the TIG welding process and heat treatment in the thermal diffusivity in samples of a duplex stainless steel UNS S32304, the knowledge of material properties, dealing with the fabrication process, is needed. The purpose is to conform to radioactive material standards. The samples were welded in pairs following an autogenous procedure using argon and nitrogen as protection gas. Then the welded pairs were heat treated at a temperature of $600{ }^{\circ} \mathrm{C}$ followed by cooling one in air and the other one in sea water. The analyses of the thermal diffusivity was measured with the use of Laser Flash Method before the thermal treatment of the samples and microstructural characterization of the material by optical microscopy. The welding areas present higher values of the thermal diffusivity than the ones found in the base metal of the samples and this result could be influenced by the increase grain size and phases imbalance.
\end{abstract}

Keywords (Palavras chaves): Aço inoxidável duplex. Soldagem TIG. Tratamento térmico. Difusividade térmica. Método Flash Laser. 


\section{Introdução}

Aços inoxidáveis duplex (AID) apresentam estrutura bifásica ferrita-austenita em proporções aproximadamente iguais a $50 \%$, o que lhes confere boa resistência à corrosão sobtensão, alta resistência à corrosão intergranular, excelentes propriedades mecânica e boa soldabilidade [1,2]. Para atingir essa proporção, é necessário o controle da composição química, temperatura de recozimento e condições de resfriamento [3]. As temperaturas elevadas geradas no processo de soldagem causam 0 desequilíbrio termodinâmico e o material busca uma condição mais estável, por meio da precipitação de novas fases, além da ferrita e austenita, levando ao desbalanceamento microestrutural. Algumas fases são previstas pelo diagrama de equilíbrio de fase para o aço inoxidável duplex UNS S32304, como carbetos $\left(\mathrm{M}_{23} \mathrm{C}_{6}\right)$, nitretos $\left(\mathrm{Cr}_{2} \mathrm{~N}\right)$ e fase sigma $(\sigma)$. A precipitação de $\mathrm{Cr}_{2} \mathrm{~N}$ é mais frequente e reduz propriedades como resistência à corrosão e tenacidade em temperaturas em torno de $600^{\circ} \mathrm{C}$ a $850^{\circ} \mathrm{C}[4,5]$. A precisão no tempo de resfriamento após a solda também garante a manutenção das propriedades desejáveis no AID, uma vez que taxas elevadas de resfriamento retarda a transformação de ferrita para austenita, promovendo a precipitação de carbetos e nitretos de cromo. Por outro lado, um resfriamento muito lento pode levar a precipitação de compostos intermetálicos [6]. Assim, faz-se necessário o estudo da difusividade térmica para o entendimento da influência dessa propriedade na cinética de transformação de fase, na qual o processo difuso do fluxo de calor oriundo da soldagem contribui para microestrutura final do material.

Visando atender as normas de segurança para utilização do material estudado no desenvolvimento de um receptáculo para transporte de materiais radioativos, neste trabalho avalia-se o comportamento da difusividade térmica do aço inoxidável duplex UNS S32304, submetido ao processo de soldagem TIG, com gás de proteção $98 \% \mathrm{Ar}+2 \% \mathrm{~N}_{2}$, e tratado termicamente a $600^{\circ} \mathrm{C}$ com resfriamento em água do mar e ar.

\section{Materiais e Métodos}

\subsection{Materiais}

Utilizaram-se tiras de aço inoxidável duplex da classe UNS S32304, com dimensões de $72,0 \mathrm{~mm}$ de largura por $1,8 \mathrm{~mm}$ de espessura e composição química, conforme especificado pelo fabricante, mostrada na Tabela 1.

\subsection{Soldagem e tratamento térmico}

Os corpos de prova foram soldados em pares, com junta tipo I, através do processo TIG autógeno, com eletrodo de tungstênio (W) dopado em $2 \%$ de tório (Th), afiado em $60^{\circ}$. Foi utilizada uma corrente pulsada de onda balanceada e frequência de aproximadamente $60 \mathrm{~Hz}$, com valor médio de $143 \mathrm{~A}$, tensão média de $13 \mathrm{~V}$ e velocidade de $35 \mathrm{~cm} / \mathrm{min}$. O gás de proteção utilizado foi uma mistura de argônio ( $\operatorname{Ar})$ e $2 \%$ de nitrogênio $\left(\mathrm{N}_{2}\right)$, com vazão de $10 \mathrm{l} / \mathrm{min}$. Foram tratadas termicamente em forno tipo mufla programável, à temperatura de $600^{\circ} \mathrm{C}$ com duração de 8 horas, seguido de resfriamento em ar e em água do mar. As amostras foram identificadas conforme tabela 2 .

Tabela 2: Identificação das amostras segundo as condições experimentais.

\begin{tabular}{|c|c|c|}
\hline Identificação & $\begin{array}{c}\text { Temperatura de } \\
\text { Tratamento }\end{array}$ & Resfriamento \\
\hline T60 & $600^{\circ} \mathrm{C}$ & Água do mar \\
\hline T68 & $600^{\circ} \mathrm{C}$ & $\mathrm{Ar}$ \\
\hline
\end{tabular}

\subsection{Microscopia óptica}

O procedimento de preparação das amostras envolveu as etapas de lixamento, com lixas de granulometrias variando de 220 a 1200 mesh, de polimento, com alumina $(1 \mu$ e $0,3 \mu)$ e de ataque eletrolítico. Para o metal base e a zona afetada pelo calor, a microestrutura foi revelada através do ataque $10 \mathrm{~N}$ de $\mathrm{KOH}, 2,5 \mathrm{~V}$ por 20s. Já a região da solda, o ataque foi realizado com solução de ácido oxálico 10\%, 6V,

\begin{tabular}{|c|c|c|c|c|c|c|c|c|c|c|c|c|}
\hline$\% \mathrm{C}$ & $\% M n$ & $\% \mathrm{Si}$ & $\% \mathrm{P}$ & $\% S$ & $\% \mathbf{C r}$ & $\% \mathrm{Ni}$ & \%Mo & Nppm & $\% \mathrm{Ti}$ & $\% \mathrm{Cu}$ & $\%$ Co & PREN* \\
\hline 0,016 & 1,4 & 0,25 & 0,023 & 0,001 & 22,2 & 3,52 & 0,255 & 1030 & 0,0041 & 0,4171 & 0,09 & 24,691 \\
\hline
\end{tabular}


durante 8s, ambos seguidos de secagem por aspersão de álcool etílico PA com evaporação estimulada por ar quente. A microestrutura foi observada através de um microscópio óptico digital com uso de lentes de aumento de 200 e 500x.

\subsection{Medidas de difusividade térmica}

As medidas de difusividade térmica foram obtidas através do método Flash Laser, que consiste em aplicar um pulso de energia radiante, intenso e curto, uniformemente sobre a face de uma pequena amostra, obtendo-se um registro do transiente de elevação de temperatura na face oposta à incidência do calor $[7,8]$. O comprimento de onda do pulso de energia utilizado foi de $10^{-5} \mathrm{~m}$, intensidade $10 \sqrt{2} \mathrm{w} / \mathrm{m}^{2}$ e diâmetro igual 2 $\mathrm{mm}$. A difusividade é calculada a partir da espessura da amostra $(L=2,101 \mathrm{~mm})$, e do tempo requerido para a temperatura da face oposta atingir a metade da

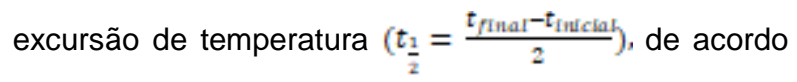
o equacionamento de Parker et al (1961) [8].

$$
\alpha_{0}=1,38 \frac{L^{2}}{\pi^{2} t_{\frac{1}{2}}}
$$

Para utilização do método é necessário fazer uso de algumas correções que levam em conta as perdas de calor que ocorrem durante a realização do experimento, bem como os efeitos de tempo finito de pulso. Utiliza-se um fator de correção, que é a razão entre o tempo de entrega do pulso de energia do laser $(\tau)$ e o tempo de subida da temperatura no material para corrigir o efeito do tempo finito de pulso. A correção também deve ser feita em relação à largura do pulso, que neste trabalho foi de 4,2 vezes maior que o permitido, influenciando na curvatura da subida $[9,10]$. Assim, o valor real da difusividade térmica deverá sofrer uma correção dada por:

$$
\alpha=\alpha_{0}\left(4,2 \frac{\tau}{t_{1}}\right)
$$

\section{Resultados e discussão}

\subsection{Microscopia óptica}

As microestruturas do aço como recebido (CR), foram obtidos por Milagre (2015) [11], Figura 1, e apresentam a estrutura característica de um aço inoxidável duplex com grãos alongados na direção da laminação, composta por ferrita (região atacada, escura) e austenita (região não atacada, clara).



Figura 1 - Microscopia Ótica da amostra CR, ataque KOH 10N. A seta indica a direção de laminação [11].

Foi possível visualizar nas zonas fundidas (ZF) das amostras soldadas e tratadas, Figuras 2 e 3 , a concentração de precipitados no interior da ferrita, função do fluxo de calor no processo de soldagem utilizado (TIG autógeno). O aumento da temperatura seguido de resfriamento em altas taxas provoca uma elevada quantidade de ferrita (fase primária na solidificação) e, por consequência, a maior precipitação de nitretos de cromo e/ou carbetos, em função da baixa solubilidade do $\mathrm{N}_{2}$ e $\mathrm{C}$ nessa fase [12]. Para os aços que contém um teor de carbono inferior a $0,02 \%$, os carbetos raramente são observados [2], assim, acredita-se que os precipitados observados corresponderiam a nitreto de cromo $\left(\mathrm{Cr}_{2} \mathrm{~N}\right)$, conforme verificado na literatura [3,13,14]. Essa formação também está relacionada com modo de resfriamento da $Z F$, que é relativamente rápido, onde a quantidade de austenita formada se distancia do valor de equilíbrio e o nitrogênio, que não consegue chegar até a austenita, precipita na ferrita como nitreto de cromo [6].

As morfologias da austenita, alotriomórfica, intragranulares e Widmanstätten, também foram identificadas, assim como observado por [3,11,13]. A morfologia Widmanstätten, geralmente resultado de uma maior restrição do processo de crescimento planar da fase [15], ficou mais evidente na amostra resfriada em água do mar, favorecida por altas taxas de resfriamento, em função dos altos gradientes de temperatura que ocorrem nos processos de soldagem. 


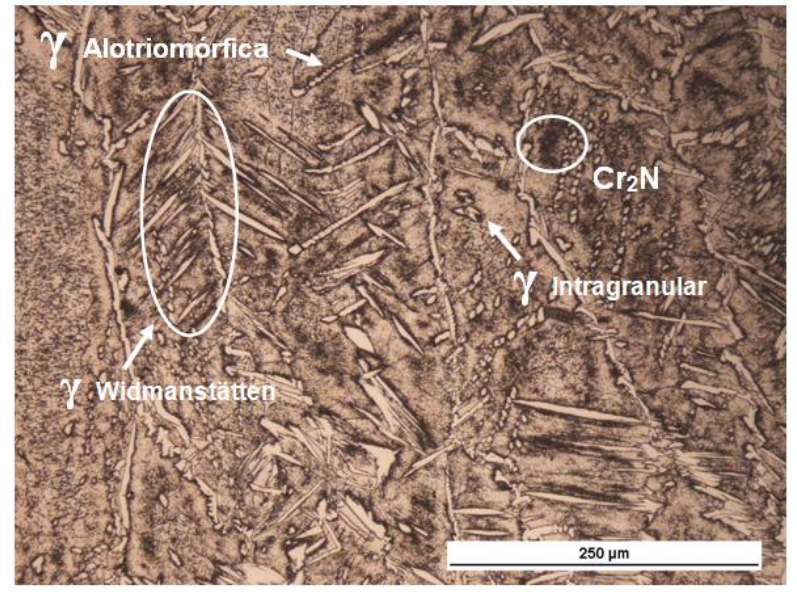

Figura 2 - Micrografia da zona fundida da amostra T60, destacando as morfologias típicas de soldagem dos AID's. Ataque ácido oxálico $10 \%$.

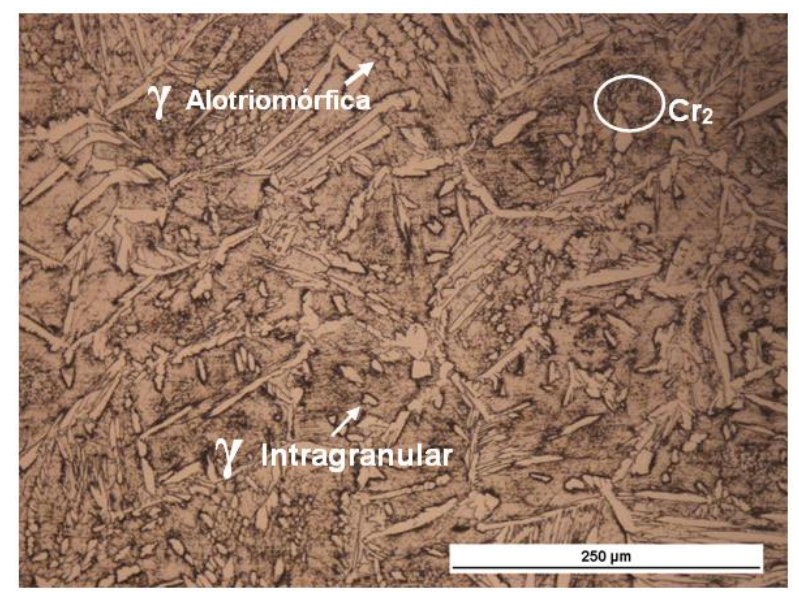

Figura 3 - Micrografia da zona fundida da amostra T68, destacando as morfologias típicas de soldagem dos AID's. Ataque ácido oxálico 10\%.

O MB, a princípio, não sofreu interferência do calor gerado no processo de soldagem, portanto suas características microestruturais estão relacionadas apenas com o tratamento térmico realizado. Não houve alterações perceptíveis na morfologia das fases, mantendo-se basicamente uma microestrutura bifásica, com grãos alongados e pequenas ilhas de austenita, conforme Figuras 4 e 5.

\subsection{Difusividade Térmica}

A difusividade térmica do material na condição como recebida foi calculada, pela relação entre a condutividade térmica e o calor específico volumétrico, através de dados fornecidos pelo fabricante, $k=17$ $\mathrm{W} / \mathrm{m} . \mathrm{K}, \quad \mathrm{Cp}=450 \mathrm{~J} / \mathrm{Kg} . \mathrm{K}$ e $\rho=7,8 \mathrm{~g} / \mathrm{cm}^{3}$, com 0 propósito de obter um parâmetro para análise, obtendose $\alpha=4,364 \cdot 10^{-6} \mathrm{~m}^{2} / \mathrm{s}$. Os dados de difusividade térmica coletados experimentalmente pelo método Flash Laser

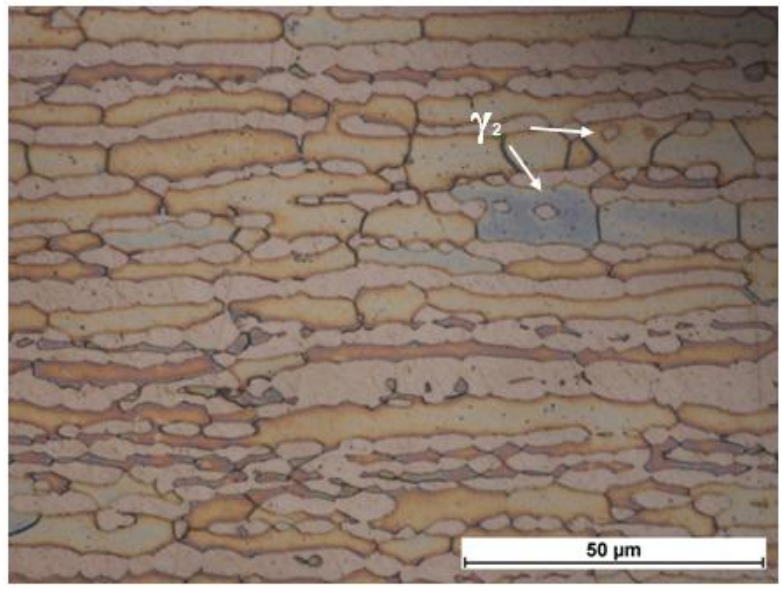

Figura 4 - Micrografia do metal base da amostra T68. Ataque $\mathrm{KOH}$ 10N. Destaque para formação de ${ }^{2}$.

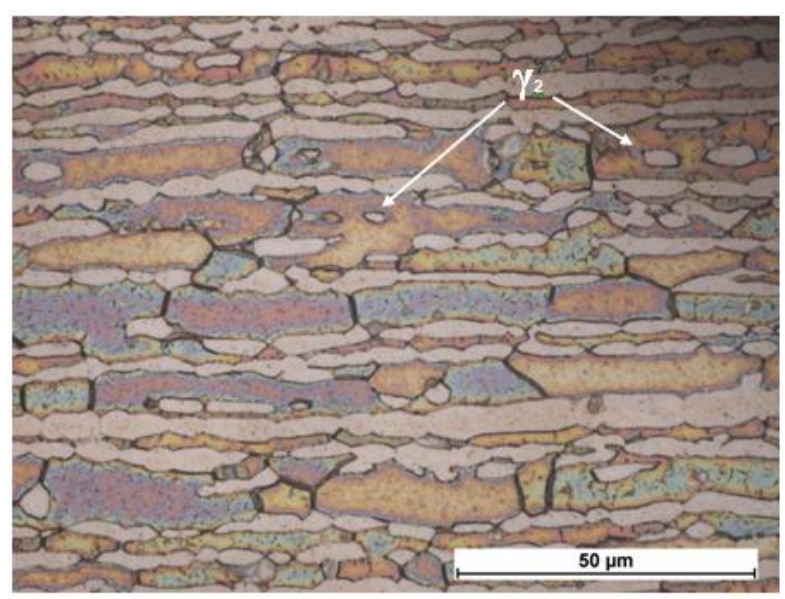

Figura 5 - Micrografia do metal base da amostra $\mathrm{T} 60$. Ataque $\mathrm{KOH}$ $10 \mathrm{~N}$. Destaque para formação de $\mathbf{2}^{2}$

foram corrigidos considerando o tempo finito de pulso, e expostos graficamente (Figura 6), considerando o erro de $5 \%$ da bancada experimental.



Figura 6 - Comparação da difusividade térmica para as amostras T60, T68 e como recebida.

Os valores de difusividade térmica das amostras apresentam o mesmo comportamento, crescentes no 
sentido metal base $\rightarrow$ zona fundida. O procedimento de soldagem promoveu um aumento da difusividade na ZF e ZAC, enquanto no $M B$, que só foi influenciado pelo tratamento térmico, não apresentou alteração significativa da propriedade. Esse comportamento pode ser influenciado pelo desequilíbrio de fases gerado pelo processo de soldagem. Com o aumento do teor de ferrita, é possível que a difusividade do material aumente, visto que para esta fase a difusividade térmica é aproximadamente 100 vezes maior que na fase austenita [16].

O aumento da difusividade também pode estar relacionado com aumento do tamanho dos grãos causado pelas altas temperaturas no processo de soldagem e tratamento térmico. Contornos de grãos e/ou de fases podem desempenhar um papel importante no controle do transporte térmico em materiais policristalinos devido à resistência térmica interfacial, ou de contorno, que resulta em uma descontinuidade de temperatura nas interfaces entre grãos na presença de um gradiente térmico [17], causando uma redução da condutividade térmica do sistema, que é diretamente proporcional a difusividade térmica. Quanto maior o tamanho dos grãos, menor o número de junções entre eles, portanto menos resistência à propagação do calor $[9,10]$. Com relação ao tratamento térmico, a amostra resfriada em ar apresentou maior difusividade térmica, o que pode ser explicado pelo aumento do tamanho dos grãos da austenita, devido ao maior de tempo para nucleação dessa fase.

\section{Conclusão}

O estudo demonstrou que o comportamento da difusividade térmica é influenciado pelo tamanho de grão e pelo desequilíbrio de fases. A elevação de temperatura associada ao processo de soldagem e tratamento térmico gerou aumento no tamanho médio dos grãos e na concentração da fase ferrita, assegurando assim o aumento da difusividade térmica na ZF e ZAC.

O valor da difusividade térmica é menor na amostra resfriada em água do mar, devido ao menor tempo de exposição à altas temperaturas.

\section{Referências}

[1] FOLKHARD, E. Welding Metallurgy of Stainless Steels. $1^{\text {a }}$ Ed. New York, 1988.

[2] GUNN, R. N. Duplex Stainless Steel: Microstructures, Properties and application, Abington Publishing. Cambridge, 1997.

[3] MACHADO, C. S. C. Perfis de tensão residual do aço duplex UNS S32304 submetido a diferentes processos de soldagem TIG e condições de tratamento térmico. Dissertação de Mestrado. Universidade Federal do Espírito Santo, Vitória, 2015.

[4] MARTINS, M. Caracterização microestrutural mecânica e resistência à corrosão do aço inoxidável super duplex ASTM A890 / A890M Grau 6A. Tese de Doutorado. Universidade de São Paulo, São Carlos, 2006.

[5] CHARLES, J. CHEMELLE, P. The history of duplex developments, nowadays DSS properties and duplex market future trends. 8th Duplex stainless steels conference, Beaune, 2010.

[6] LIPPOLD, J.C.; KOTECKI, D.J. Welding Metallurgy and Weldability of Stainless Steel. Hoboken, New Jersey, USA: John Wiley \& Sons, 2005.

[7] PARKER, W. J.; et al. Flash Method of Determining ThermalDiffusivity, Heat Capacity and ThermalConductivity. U.S. Naval Radiological Defense Laboratory, San Francisco, 1961.

[8] GROSSI, P. A. et al. Metodologia para a estimativa da incerteza de medição da difusividade térmica pelo método do Flash Laser. In: Anais do $8^{\text {o }}$ Congresso Iberoamericano de Engenharia Mecânica, Cusco. 2007.

[9] ALMEIDA, L. C. Estudo da Difusividade Térmica em Amostras Cerâmicas de $\mathrm{Ca}_{2-\mathrm{x}} \mathrm{Y}_{\mathrm{x}} \mathrm{MnReO}_{6} \operatorname{com} \mathrm{x}$ entre 0 e 0,3. Dissertação de Mestrado. Universidade Federal do Espírito Santo, Vitória, 2013.

[10] ROUVER, A. N. Determinação da influência do contorno de grão na medida de difusividade térmica em cerâmicas supercondutoras $\mathrm{YBa}_{2} \mathrm{Cu}_{3} \mathrm{O}_{7}-\delta$. Dissertação de Mestrado. Universidade Federal do Espírito Santo, Vitória, 2013. 
[11] MILAGRE, M. X. Perfis de tensão residual do aço duplex UNS $\$ 32304$ submetido a processo de soldagem TIG e diferentes condições de tratamento térmico de têmpera. Dissertação de Mestrado. Universidade Federal do Espírito Santo, Vitória, 2015.

[12] SILVA, S. D. Efeito do tratamento térmico póssoldagem no comportamento em corrosão e nas propriedades mecânicas do aço inoxidável duplex UNS S32304. Dissertação de mestrado. Universidade Federal do Espírito Santo, Vitória, 2010.

[13] LONDOÑO, A. J. R. Precipitação de Fases Intermetálicas na ZAC de Soldagens Multipasse de Aços Inoxidáveis Duplex. Tese de Doutorado. Universidade de São Paulo, São Paulo, 2001.

[14] PALÁCIO, F. O. Estudo do efeito de parâmetros do processo GTAW pulsado na resistência à corrosão intergranular e por pite do aço inoxidável duplex UNS S32304. Dissertação de mestrado. Universidade Federal do Espírito Santo, Vitória, 2011.

[15] KOU, S., Welding metallurgy, 2nd edition, 2003, John Willey and Sons, Inc., USA, ISBN 0-47143491-4.

[16] LOURENÇO, J. M. et al. Processamento por plasma de amostras de aço IF utilizando diferentes montagens de eletrodos. Holos, v.1, p.65-70, 2005.

[17] YANG, H. S., et al. Interfacial Thermal Resistance in Nanocrystalline Yttria-Stabilized Zirconia. Acta Materialia, v. 50, n. 9, p. 2309-2317, 2002. 